\title{
Multilinguales
}

\section{Enseignement de l'oral à l'université en Algérie et Cadre Européen Commun de Référence}

Teaching oral expression at university and

$$
\begin{aligned}
& \text { the common European framework of reference } \\
& \text { تعليمية } \\
& \text { الشفوي } \\
& \text { في }
\end{aligned}
$$

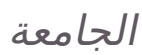

$$
\begin{aligned}
& \text { والإطار } \\
& \text { المرجعي } \\
& \text { الأوروبي } \\
& \text { المشترك }
\end{aligned}
$$

\section{Hamida Doulate Serouri}

\section{(2) OpenEdition \\ Journals}

Édition électronique

URL : http://journals.openedition.org/multilinguales/755

DOI : $10.4000 /$ multilinguales.755

ISSN : 2335-1853

\section{Éditeur}

Université Abderrahmane Mira - Bejaia

\section{Référence électronique}

Hamida Doulate Serouri, «Enseignement de l'oral à l'université en Algérie et Cadre Européen Commun de Référence- », Multilinguales [En ligne], 7| 2016, mis en ligne le 31 décembre 2016, consulté le 17 septembre 2019. URL : http://journals.openedition.org/multilinguales/755 ; DOI : 10.4000/ multilinguales.755 
Ce document a été généré automatiquement le 17 septembre 2019.

\section{(C) $10 \Theta$}

Multilinguales est mise à disposition selon les termes de la Licence Creative Commons Attribution Pas d'Utilisation Commerciale - Pas de Modification 4.0 International 


\section{Enseignement de l'oral à l'université en Algérie et Cadre Européen Commun de Référence}

Teaching oral expression at university and

$$
\begin{aligned}
& \text { the common European framework of reference } \\
& \text { تعليمية }
\end{aligned}
$$

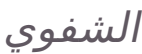

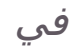

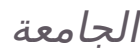

$$
\begin{aligned}
& \text { والإطار } \\
& \text { المرجعي } \\
& \text { الأوروبي } \\
& \text { المشترك }
\end{aligned}
$$

Hamida Doulate Serouri

Le développement des compétences de l'oral est reconnu aujourd'hui comme primordial pour la maîtrise d'une langue étrangère (Gadet, Le Cunff et Turco : 1998; Garcia-Debanc et Delcambre, 2002). Dans le système d'enseignement, en Algérie, l'oral en langues étrangères est programmé dans les deux cycles, primaire et secondaire, et 
en licences de langues étrangères. Par ailleurs, en licences de langues L/LMD ${ }^{1}$, de trois (3) ans,

un nouveau statut ${ }^{2}$

lui accorde une place plus importante que celle qui lui était réservée en licence dite

" classique »

Dans les nouveaux canevas et programmes, des modules d'un volume horaire de plusieurs heures, intégrés dans des unités d'enseignement fondamental, lui sont consacrés pendant les deux premières années.

Nous avons jugé pertinent de nous interroger sur les démarches didactiques qui pourraient contribuer à développer les compétences de production et d'interaction orales des étudiants du tronc-commun de la licence de français, à travers quelques-uns des principes de l'approche actionnelle. Nous comptons apporter des éléments de réponses à quelques questions de fond: Quels genres d'activités peut-on proposer aux étudiants? Quelles sont les tâches à réaliser? Quels sont les critères d'évaluation à retenir ? Comment remédier aux lacunes des apprenants.

\section{Méthodologie}

Nous formulons l'hypothèse qu'un choix approprié d'activités et de thèmes à travailler en interactions orales pourrait stimuler les étudiants et les amener à participer à la communication orale, à débattre, en un mot, à réaliser des tâches analogues à celles de la vie réelle.

5 Précisons que la notion de " tâche » est à comprendre au sens de D. Coste $(2009: 15)$ : « La notion de tâche renvoie à une action finalisée, avec un début, un achèvement visé, des conditions d'effectuation, des résultats constatables (réparer une machine, remplir un formulaire, (...)».

6 Dans la perspective actionnelle, préconisée par le Cadre Européen Commun de Référence (Conseil de l'Europe, 2001), cette conception s'explique par le statut d'« acteur social » qu'elle confère à l'apprenant ou à l'utilisateur de la langue.

7 Dans cette perspective, l'apprentissage et la communication sont envisagés comme des actions qui se réalisent à travers des activités langagières qui correspondent à des actes de parole (se présenter, demander une information, remercier quelqu'un, etc.), à des genres de discours (débats, conférences, genres conversationnels, etc.) :

8 Dans cette perspective, l'apprentissage et la communication sont envisagés comme l'accomplissement de tâches qui s'exécutent à travers des activités langagières (conseil de l'Europe, 2001: 15). Chacune d'elles se réalise «dans des circonstances et un environnement donnés, à l'intérieur d'un domaine d'action particulier» (idem), comme l'explique Tagliante :

Cette approche permet d'élaborer des activités d'apprentissage qui traitent de la compétence langagière générale (interaction, production, réception, médiation), dans des domaines précis (personnel, public, éducationnel, professionnel), en réalisant des tâches communicatives dans des situations caractérisées par des lieux, des organismes, des acteurs, des objets, des événements. (Tagliante, 2007a : 32)

Pour vérifier notre hypothèse, nous avons mené, au cours de l'année universitaire 2014-2015, une expérience auprès des étudiants de $2^{\text {ème }}$ année de licence de français 
langue étrangère. Le test a été réalisé durant les séances de Travaux Dirigés (TD) du module de compréhension et expression orales.

Nous avons constitué un groupe de trente étudiants dont la présence aux TD est régulière, et dont le niveau de langue en français est hétérogène. Les tâches qui leur étaient demandées s'inscrivaient dans des domaines différents: personnel, professionnel, éducationnel. Elles étaient à réaliser à travers des activités de jeux de rôles, de simulation et d'interaction. Elles se référaient aux différentes catégories de tâches proposées dans le Cadre Européen Commun de Référence (Conseil de l'Europe, 2001). Il s'agit de huit activités langagières pour l'oral et l'écrit qui relèvent de la réception, de la production, de l'interaction et de la médiation dont le CECR souligne l'intérêt :

Afin de réaliser des tâches de communication, les usagers de la langue doivent

s'impliquer dans des activités langagières communicatives. De nombreuses activités communicatives telles que la conversation ou la correspondance, par exemple, sont interactives, c'est-à-dire que les participants sont tour à tour locuteur(s)/ scripteur(s) et destinataire(s). (Conseil de l'Europe, $2001: 48$ )

11 Nous avons choisi deux types d'activités d'interaction orale, à savoir le débat et les activités théâtrales.

$12 \mathrm{~L}$

e débat est un genre de discours oral qui se caractérise par une discussion sur une question qui suscite des prises de position contradictoires. D'après Dolz et Schneuwly, les apprenants sont familiers de ce type d'activité parce que courant dans les émissions radiophoniques et télévisuelles :

Les raisons en faveur d'un travail sur le débat en public, à différents moments de la scolarité obligatoire, sont nombreuses. Les élèves vivent dans une société de persuasion dans laquelle les médias essaient systématiquement d'influencer

l'opinion des citoyens [...]. Lorsqu'ils interviennent dans un débat en classe, ils se préparent à participer à la vie sociale [...], ils découvrent certains ressorts de

l'argumentation et s'arment ainsi contre certains effets manipulatoires. $(2014: 163)$

\section{Ces représentationsa priori}

des situations argumentatives en public chez les apprenants expliqueraient que ce type d'activité soit fréquemment sollicité par les enseignants de langue.

Quant aux activités théâtrales, elles avaient déjà été introduites dans les méthodes conventionnelles, notamment les méthodes SGAV et communicative-cognitive dont un des principes de base était de ne pas séparer la pratique linguistique de ses conditions d'emploi ordinaire.

Dans Dictionnaire de didactique du français langue étrangère et seconde, on souligne l'intérêt de ce genre oral pour les apprenants :

Le théâtre dans la classe de FLE offre les avantages classiques du théâtre en langue maternelle : apprentissage et mémorisation d'un texte, travail de l'élocution, de la diction, de la prononciation, expression de sentiments ou d'états par le corps et par le jeu de la relation, expérience de la scène et du public, expérience du groupe et écoute des partenaires, approche de la problématique acteur/personnage, être/ paraître, masque/rôle (Cuq, 2003 : 237).

L'activité théâtrale semble incontournable en enseignement du FLE. Selon le Cadre Européen Commun de Référence, ces pratiques didactiques favorisent l'apprentissage de la langue étrangère: "En ce qui concerne les tâches ou activités en tant que moyens pour planifier et mener à bien l'enseignement et l'apprentissage, on peut donner s'il y a lieu 
l'information concernant les types de tâches, par exemple, simulations, jeux de rôle, interactions en classe, etc. (Conseil de L'Europe, $2001: 47$ ).

Quant au choix des thèmes, au lieu de les imposer aux apprenants comme c'est souvent le cas $^{4}$, nous avons opté pour des thèmes négociés avec les étudiants pour les motiver davantage et développer leurs sens de l'initiative et de l'autonomie, indispensables à l'apprenant/acteur social :

Le développement de l'autonomie implique qu'on donne aux apprenants une certaine latitude quant au choix du contenu et de la méthode. L'enseignant est habitué à présenter et à exploiter un texte unique [...]. Il lui faut un certain courage pour livrer, au moins en partie, à la négociation les matériels et les activités pédagogiques. (Sheils, $1993: 28$ ) Pour le débat, trois thèmes ont été retenus : « les femmes dans des métiers d'hommes », « la valeur de l'argent » et les « avantages et inconvénients des réseaux sociaux ».

Quant à l'activité théâtrale, elle a consisté en de courtes scènes autour de «la pausecafé à l'hôpital ».

Pour la collecte des données, nous avons enregistré les activités d'interactions orales en bandes vidéo que nous avons transcrites intégralement selon les conventions de transcription du GARS ${ }^{5}$. Cet outil nous a permis de mesurer les différents aspects de la communication orale verbale et non-verbale et d'étudier les tours de parole.

\section{Description des activités d'interaction orale}

\section{Le débat}

Nous avons analysé les données issues de l'observation de la réalisation de trois tâches qui relèvent de l'interaction orale et qui ont permis aux apprenants d'exprimer leurs opinions, de prendre position et de justifier leurs points de vue. La première, un débat d'environ 8 minutes sur «les femmes dans des métiers d'hommes ", a été organisée suite à une activité de compréhension orale d'un reportage télévisé qui traitait de l'image de la femme dans des professions habituellement réservées aux hommes ${ }^{6}$. Deux groupes d'étudiants de 5 et 3 membres, dont les avis étaient contradictoires, ont confronté leurs arguments.

La seconde est un autre débat d'environ 4 minutes, entre un étudiant et un groupe de quatre de ses camarades, sur « la valeur de l'argent».

La troisième est une discussion d'environ 6 minutes, menée par un groupe de quatre étudiantes autour des "avantages et inconvénients des réseaux sociaux " (Facebook, Twitter, Skype, etc.).

Il est à retenir que ces activités relèvent tantôt de l'oral improvisé, tantôt de l'oral préparé, et que leur réalisation a impliqué l'exécution de différentes tâches et de différents actes de paroles: donner et défendre un point de vue, étayer son argumentation par des exemples, réfuter un argument en utilisant les outils linguistiques appropriés, etc. 


\section{L'activité théâtrale} parce qu'il implique plusieurs acteurs autour d'un enjeu: "Une interaction donnée entre deux ou plusieurs locuteurs engagés dans l'échange est un événement de communication constitué par ce jeu d'influence de paroles et des actes sur les paroles et les actes des locuteurs " (Beacco, 2007 : 123). improvisé car, après s'être concertés sur le choix du thème, ils intervenaient librement, sans avoir recours à leurs carnets de notes. D'autres ont opté pour l'oral préparé parce qu'il leur permettait d'organiser leurs idées avant d'en débattre, d'apprendre par cœur quelques expressions et de choisir leurs arguments avant de prendre la parole. Ces choix montrent quelque peu la diversité des profils des étudiants.

Les étudiants ont participé activement au premier débat, « les femmes dans des métiers d'hommes ", en essayant de donner leurs points de vue et de prendre position par rapport à ce thème qui a rapidement généré un conflit entre eux. Notons que le sujet traite d'un thème auquel les étudiants ont déjà été confrontés dans la vie réelle et qui relève de la question maintes fois débattue de la place de la femme dans la société. Ce 
qui est intéressant à relever est dans la posture adoptée par les uns et les autres : parmi les arguments les plus contradictoires entendus dans les débats sociaux, ils ont choisi les leurs et se les opposent dans un débat organisé.

Extrait $1:$ «les femmes dans des métiers d'hommes »

L1 :

euh on est pour le travail des femmes dans des métiers masculins euh parce que de nos jours la femme euh s'est =imposée dans la société même euh elle s'est battue pour euh avoir une égalité avec l'homme ++ et notamment dans des métiers qui préservaient aux + aux =hommes on trouve parmi ces métiers on trouve des femmes qui occupent des + des postes classiquement réservés aux =hommes comme euh + Angela Merkel qui / L2:

elle prend le métier de l'homme comme par exemple tel que les ingénieurs en bâtiment ou + ou bien en génie civile euh l'avantage de ce métier + que la femme est =égale à

l'homme

L3 :il n'y a pas de travail d'homme et de travail de femmes +

L4 :××x elle ne peut pas exercer des métiers pareils parce qu'elle est fragile

L7 :mais

je pense + elle perdrait sa féminité elle sera méprisée et non respectée par les gens/

L8 :mais notre euh nos traditions ne permet pas la femme de : /

L1 :

mais à condition sans atteindre sa féminité je suis pour le travail des femmes dans des métiers d'hommes/

L4 :impossible c'est• impossible + c'est• impossible tu peux faire /

L6 :

parce que le contraire + ce genre de travail ××x elle va perdre sa féminité elle n'aura pas

L8 :non c'est pas tous les domaines /

le temps de s'occuper de son foyer

L7 :aussi elle négligerait sa vie privée

L4:

oui ses enfants son + son travail domestique + elle peut pas faire les deux tâches + un travail dur et faire euh

L'extrait ci-dessus donne un aperçu des tours de parole. Il apparaît clairement que les interactants étaient engagés dans l'interaction orale. Certains ont pris l'initiative et ont monopolisé soit volontairement, soit involontairement, la parole (L1 et L4). Nous avons enregistré 67 tours de parole des étudiants impliqués dans l'activité, en écartant les paroles inaudibles des participants qui parlent tous à la fois (L0) et l'intervention de l'enseignante (E) qui a joué le rôle d'animatrice (tableau 1) :

Tableau $\mathrm{N}^{\circ}$ 1: Débat $\mathrm{N}^{\circ} 1$

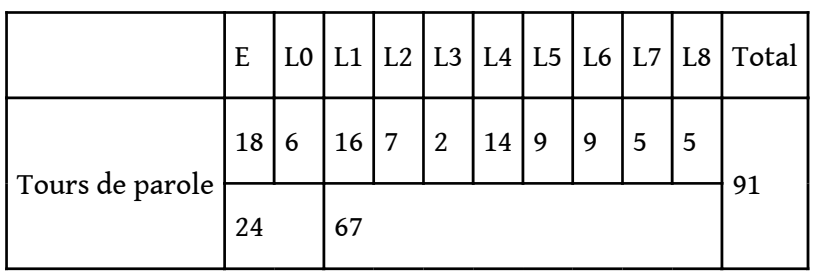


qui concerne la gestion de l'alternance des tours de parole, nous avons remarqué la présence d'interruptions très courtes dans la plupart des cas, ce qui a permis aux interactants de prendre part à l'échange. De même, les chevauchements à valeur coopérative ont eu pour but d'assurer la continuité thématique (négation et réfutation des propos d'autrui ou étayage de l'idée) :

L1:

mais à condition sans atteindre sa féminité je suis pour le travail des femmes dans des métiers d'hommes/

L4 :impossible c'est• impossible + c'est• impossible tu peux faire / (...)

L4 :oui généralement les femmes euh

L7 :elle sera guidée par ses sentiments

37 Cela indique que chaque participant a pris en compte les propos des autres, et qu'il est à la fois locuteur et destinataire comme préconisé par le CECRL (Conseil de l'Europe, 2001 : 48). Ainsi, nous pouvons considérer le travail coopératif qui s'est effectué entre les apprenants comme une stratégie qui leur a permis de prendre le risque de s'engager dans une communication: "L'apprenant s'investit davantage, gagne en respect de soi et en assurance, et stimulé par l'ambiance "socio-affective" conviviale de la classe, il incline plus volontiers à prendre des risques " (Sheils, $1993: 8$ ).

Nous avons également constaté que la plupart des participants utilisaient la langue avec aisance, sans faire des pauses pour chercher leurs mots, et étaient capables de justifier leur point de vue :

L4 :××x elle ne peut pas exercer des métiers pareils parce qu'elle est fragile

L7 :mais

je pense + elle perdrait sa féminité elle sera méprisée et non respectée par les gens/

Leurs propos sont clairs et illustrés par des exemples:

L2 : elle prend le métier de l'homme comme par exemple tel que les ingénieurs en bâtiment ou + ou bien en génie civile euh l'avantage de ce métier + que la femme est =égale à l'homme

Selon Cuq et Gruca, qui insistent surtout sur l'aisance dans l'expression et la fluidité, ce sont là des indicateurs de la maîtrise de l'oral en FLE. Pour eux, en effet, "la maîtrise de la langue orale est souvent estimée à travers sa fluidité dans des échanges primaires : habileté à parler de façon continue, sans arrêt sur les mots, mais sans forcément employer des formulations élaborées» (2005 : 179). Nous pouvons donc en déduire que c'est le cas de nos étudiants.

Par rapport à l'échelle élaborée par le CECRL, les étudiants qui ont participé à ce débat peuvent être classés au niveau $B 2^{7}$ (niveau de l'utilisateur qui «peut communiquer »), car l'

«utilisateur indépendant B2: Peut communiquer avec un degré de spontanéité et d'aisance [...]. Peut s'exprimer de façon claire et détaillée sur une grande gamme de sujets, émettre un avis sur un sujet d'actualité et exposer les avantages et les inconvénients de différentes possibilités » (Conseil de l'Europe, $2001: 25$ ).

\section{Débat $n^{\circ} 2$}

Les participants au deuxième débat intitulé "la valeur de l'argent ", thème souvent débattu dans la vie réelle, ont tenté de présenter leurs points de vue en recourant à des exemples simples :

Multilinguales, 7 | 2016 
Extrait $2:$ : la valeur de l'argent »

L6: euh à mon avis l'argent a une grande importance et une grande valeur aussi c'est =une base plus $\times$ elle nous permet d'avoir une vie plus stable je pense qu'elle nous donne envie et on serait capable de tout faire + enfin et sans aucun doute l'argent a une grande valeur dans la vie ////

L1 : oui : mais il faut travailler pour avoir de l'argent il faut pas rester les bras croisés

L2 : les bras croisés + la valeur + l'argent a une valeur mais avec des limites c'est-à-dire pas euh + pas faire n'importe quoi ou travailler les =interdits pour avoir de l'argent + on est contre cette idée

L7 : à votre avis : l'argent est importante très importante ou un peu importante

L2 : très =importante

L0 : (rire)

L2 : mais comme on a dit + avec des limites + avec des principes bien sûr

L4: on doit savoir comment les gérer

L5 : donc selon vous l'argent est un =constituant du bonheur c'est pas le bonheur

L2 : bien sûr + c'est pas le bonheur + le bonheur c'est un truc intouchable c'est la satisfaction de soi-même

L5 : donc vous ne pouvez pas dire que l'argent c'est l' bonheur

Cet échange nous a permis d'enregistrer 47 tours de parole. Nous avons également remarqué que tout le groupe-classe s'intéressait au sujet: chaque apprenant suivait attentivement la réaction des autres par rapport au sujet; d'où la participation spontanée de certains étudiants (L4 et L7) pour atténuer le conflit (tableau 2) :

Tableau $\mathrm{N}^{\circ} 2$ : Débat $\mathrm{N}^{\circ} 2$

\begin{tabular}{|l|l|l|l|l|l|l|l|l|l|l|}
\hline & E & L0 & L1 & L2 & L3 & L4 & L5 & L6 & L7 & Total \\
\hline \multirow{2}{*}{ Tours de parole } & 6 & 2 & 2 & 23 & 1 & 3 & 13 & 1 & 4 & \\
\cline { 2 - 10 } & 8 & & \multicolumn{1}{|l|}{47} & & & & & & \\
\end{tabular}

Il apparait nettement que certains locuteurs (L2 et L5) monopolisaient la parole et prenaient position. Le discours produit est simple, cohérent, caractérisé par des énoncés brefs dans la plupart des tours de parole :

L2 : il fait le bonheur

L5 : ah + non donc c'est pas le bonheur

L2 : il fait le bonheur

L7 : c'est pas le bonheur mais il peut

Nous avons pu relever des interruptions et des chevauchements dans les prises de parole. Cela confirme que les locuteurs s'écoutaient mutuellement et réagissaient aux propos les uns des autres. Cette prise en compte de la parole d'autrui est marquée par des indices tels que la négation :

L2 : il fait le bonheur / L5: ah + non donc c'est pas le bonheur), ratification qui se produit entre locuteurs de même avis (L1: oui : mais il faut travailler pour avoir de l'argent il faut pas rester les bras croisés / L2 : les bras croisés + la valeur + l'argent a une valeur mais avec des limites c'est-à-dire pas euh. 
Ainsi, le travail interactif est indéniablement coopératif. Les interactants s'entreaidaient, sans laisser le silence s'installer. Outre la stratégie coopérative, ils ont adopté celle de compensation (le bonheur c'est un truc intouchable).

Sur le plan linguistique, les participants ont montré leur capacité à construire des énoncés cohérents en relation avec le thème, à exprimer une opinion - à mon avis, je pense, à votre avis, selon vous, personnellement) - et à donner des explications - des limites c'est-à-dire pas euh + pas faire n'importe quoi -.

Sur l'échelle du CECRL, ils peuvent être classés au niveau B1, niveau de l'usager qui « peut produire un discours simple et cohérent sur des sujets familiers et dans ses domaines d'intérêt. Peut raconter un événement, une expérience ou un rêve, décrire un espoir ou un but et exposer brièvement des raisons ou explications pour un projet ou une idée » (Conseil de l'Europe, $2001: 25$ ).

\section{Débat $n^{\circ} 3$}

50 Le troisième débat, qui a porté sur « les avantages et les inconvénients des réseaux sociaux ", a fortement suscité l'intérêt des étudiants qui ont échangé leurs opinions sans les opposer. En effet, pour les participants à la discussion, les réseaux sociaux sont des moyens de communication qu'ils ne peuvent pas éviter malgré leurs inconvénients :

1 Extrait $3:$ «les avantages et les inconvénients des réseaux sociaux »

L1 :

social donc oui le Facebook il rapproche les gens des quatre coins du monde on peut parler avec des personnes même + en chine mais il a des =inconvénients il rapproche les plus les plus loin et euh éloigne les plus proches + c'est-à-dire nos parents nos frères nos sœurs on est avec eux avec nos corps mais pas avec nos têtes + on est ailleurs même si on est à table même si on est entre famille tout le monde + chacun a son Smartphone

dans la main euh il a des =avantages bien sûr car euh il partage des ++ des =infos sur tout + sur tout le monde des --- [...]mais euh avec ces moyens de communication même si une femme par exemple accouche deux enfants à + en Indonésie on peut savoir tout ${ }^{\circ}$ suite c'est + c'est =un moyen rapide euh mais + ça prend du temps quand même + sans se sans se rendre compte on peut être assis jusqu'à cinq heures six heures sur notre euh truc à regarder les vidéos à parler à + à faire euh à faire du tchatche avec des gens même avec nos =amis ++ moi personnellement avec euh avec mon Smartphone je suis bronchée $\mathrm{H}$ vingt-quatre sur Facebook je suis tout le temps en ligne c'est-à-dire si on

cherche on me trouve + c'est devenu un (rire) un truc euh on peut dire accro

L7 : le Facebook est un réseau social qui permet de + euh communiquer avec n'importe qui à n'importe quel moment euh mais pour moi c'est =une perte de temps /

L1 : (étonnement puis rire) c'est pas vrai elle est tout le temps connectée mais c'est pas vrai elle est $\mathrm{H}$ vingt-quatre connectée /

$\mathrm{E}:$ c'est son point de vue + elle dit qu'elle perd son temps

L7 : oui parce que je suis $\mathrm{H}$ vingt-quatre connectée euh pour moi c'est =une perte de temps parce que je suis $\mathrm{H}$ vingt-quatre connectée + il prend tout mon temps

L1 : yak

L7 : (rire) --- j'ai pas le temps d'être avec mes parents mes sœurs euh $\times \times \times$ avec mes =amis / 
L1 : on raconte de tout et de rien + c'est-à-dire on refait ×x× genre euh t'as remarqué ce matin on a fait ça on a on a parlé de : + on a fait un débat sur le Facebook + flane a dit flana a dit + et on parle + si on + par exemple à cinq heure chacune part chez elle alors qu'est-ce que vous avez préparé pour le dîner qu'est-ce que + on se change de recettes + on se change de: on se raconte des films ratés si quelqu'un a raté $\times \times x$ donc on fait des =exposés + on fait un groupe généralement à trois + un groupe. Nous avons enregistré 25 tours de parole sans éliminer les interventions de certains étudiants (L2, L4, L5) qui ont prêté attention à la discussion de leurs camarades directement impliqués dans le débat (tableau $\left.{ }^{\circ} 3\right)$ :

Tableau $\mathrm{N}^{\circ} 3$ : Débat $\mathrm{N}^{\circ} 3$

\begin{tabular}{|l|l|l|l|l|l|l|l|l|l|l|}
\hline & E & L0 & L1 & L2 & L3 & L4 & L5 & L6 & L7 & Total \\
\hline \multirow{3}{*}{ Tours de parole } & 10 & 2 & 12 & 3 & 3 & 1 & 1 & 1 & 4 & \\
\cline { 2 - 10 } & 12 & \multicolumn{1}{|l|}{25} & 37 \\
& 12 & & & & & & & \\
\hline
\end{tabular}

La locutrice L1, qui s'exprimait en langue étrangère sans difficulté, qui utilisait la langue avec souplesse (elle a ouvert la discussion et pris la parole 12 fois), peut être classée en C1 d'après les indicateurs du CECRL :

Utilisateur expérimenté $\mathrm{C} 1$ : Peut s'exprimer spontanément et couramment sans trop apparemment devoir chercher ses mots. Peut utiliser la langue de façon efficace et souple dans sa vie sociale, professionnelle ou académique. Peut s'exprimer sur des sujets complexes de façon claire et bien structurée et manifester son contrôle des outils d'organisation, d'articulation et de cohésion du discours. (Conseil de l'Europe, $2001: 25$ )

En nous référant à Tagliante $(2007 \mathrm{~b}: 48-50)^{8}$, nous avons classé les compétences développées à l'oral en :

- compétences linguistiques: lexique et vocabulaire liés à la thématique (social - facebook réseau - communiquer), expression de la subjectivité (pour moi, personnellement), grammaire de l'oral (vocabulaire approximatif «truc», répétition » ce matin on a fait ça on a on a parlé de :+ on a fait un débat ", principe de l'économie « des infos »;

- compétences sociolinguistiques (introduction des termes et expressions du dialecte arabe : $y a k^{9}$, flane $^{10}$, flana $\left.^{11}\right)$;

- compétences pragmatiques qui englobent la compétence discursive (organiser des phrases cohérentes, structurer son discours) et la compétence fonctionnelle (reconnaître et produire des énoncés liés aux fonctions de la langue).

Enfin, au cours de la réalisation de la tâche, nous avons constaté que les participants mobilisaient différentes ressources stratégiques: la planification en préparant les propos et quelques exemples, l'exécution en demandant de l'aide et la traduction du terme en langue cible et la compensation par des expressions telles que "truc, quelque chose, genre ", l'autocorrection "social donc oui le Facebook [...] par exemple accouche deux enfants à + en Indonésie).

C'est, selon Tagliante (2007b), l'illustration de la gestion maîtrisée des tours de parole par les étudiants : 
En interaction, la planification d'une production (sa préparation, son adaptation, voire sa répétition), l'exécution parfois la mise en œuvre de stratégies d'évitement ou de contournement, par exemple lorsqu'on paraphrase si on ne connaît pas le mot exact, il est possible de gérer les tours de parole, en mobilisant l'attention, en coopérant, en demandant de l'aide, en prenant l'initiative ou, au contraire, en laissant des silences s'installer à dessein, la compensation en utilisant les expressions apprises par cœur, la remédiation si la communication est mal passée, l'autocorrection lorsqu'on relève soi-même ses erreurs et qu'on les corrige immédiatement. (Tagliante, 2007b : 39 - 40).

\section{Activités théâtrales}

Pour ce qui est des activités théâtrales, nous avons mis l'accent sur la correction phonétique (intonation, rythme, accent) et sur la gestualité car les interactions orales ne se limitent pas à la communication verbale mais s'étendent au non verbal. C'est pourquoi, dans le Cadre Européen Commun de Référence pour les langues, on insiste sur le fait de prendre en compte la communication orale dans tous ses aspects (verbal et nonverbal) :

La communication non-verbale est également décrite dans tous ses aspects. Le Cadre européen commun décrit les gestes et les actions (désignation d'un objet du doigt ou de la main), le comportement paralinguistique (le langage du corps et du regard, l'utilisation des onomatopées, la qualité de la voix et du ton utilisés.

(Tagliante, 2007b : 40)

Dans les trois activités, les participants ont adapté leur voix et leurs gestes à la situation de communication. D'abord, au cours de la réalisation de la simulation projective "Pause-café à l'hôpital », les participants ont eu une attitude réactive nonverbale pour renforcer leur discours oral :

Extrait 4 : « pause-café à l'hôpital »: Scène 1 : le professeur cherche les infirmiers

$1 \mathrm{~L} 1$ : (en tournant dans la salle, il fait des gestes avec les mains d'un air énervé) j' n'arrive pas à supporter ces ces =infirmiers là toujours en train de s'échapper + oh mon dieu $\times \backslash$ ils vont me tuer ces =infirmiers $\uparrow$

$2 \mathrm{~L} 2$ : oui docteur

$3 \mathrm{~L} 1$ : oui docteur ah oui docteur : $\backslash$ (lève les bras et parle de manière ironique) (rire des autres) (les mains sur les hanches) où vous =étiez /

$4 \mathrm{~L} 2$ : toujours là monsieur (geste avec les mains)

$5 \mathrm{~L} 1$ : ah : ah bon + maintenant [metnõ] (en s'adressant au L3) j'ai j'ai une opération à faire et parmi vous (geste avec l'index de la main droite et main gauche sur la hanche gauche) je n'ai pas besoin de personnes sauf celui-là viens (quitte le lieu)

$6 \mathrm{~L} 3$ : ok monsieur (rejoint L1)

Nous avons essayé de décrire, entre parenthèses, les gestes, les mouvements et les positions du corps de chaque apprenant-acteur.

Quant à l'intonation, les participants en utilisaient une fortement montante pour exprimer la colère (ils vont me tuer ces=infirmiers $\uparrow$ ), légèrement montante pour l'interrogation (où vous =étiez $/$ ), fortement descendante pour l'étonnement (oh ya lala $\downarrow$ ).

\section{ironique, (c'est $d$-c'est $d$ - c'est de moi que vous parlez de moi de moi). Nous avons également} remarqué le recours à la langue maternelle (ah non ya lala ${ }^{12}$ sauf si on est déjà fou). 


\section{Scène $1:$ deux femmes se croisent}

L1 : (marche et se rapproche du L2) (un bout de papier dans la main droite) (la main droite sur la bouche) bonjour $\uparrow$

L2 : (hoche la tête de haut en bas) (la main gauche dans la poche) bonsoir /

L1 : (s'éloigne un peu) (le bout de papier dans la main sur la bouche) bonsoir $\backslash+$ (se rapproche un peu) bonjoir [bõzwas] /

L2 : bonsour [bõsur] \

$\mathrm{L} 1:$ je peux vous $=$ aider $\uparrow$

L2 : vous =avez besoin d'un renseignement /

L1 : (s'éloigne un peu puis se rapproche) (tapotement des doigts de la main : le pouce et le majeur) psitt psitt vous m'entendez $\uparrow$

L2 : (les mains dans les poches) vous =êtes sûr (ouvre et ferme la bouche) vous =êtes sûr que ça va /

L1 : vous parlez français vous $\uparrow$ (le bout de papier dans la main sur la bouche)

L2: do you speak french (hoche la tête)

L1: do you speak french $\uparrow$ (d'un air ironique) + a donde vas $\uparrow$

Les participantes avaient planifié à l'avance l'activité (choix des répliques, distribution des rôles, préparation préalable) et n'avaient pas besoin de leurs notes. Elles parlent spontanément, avec aisance. L'expression corporelle (signalée entre parenthèses) a été importante pour l'exécution de cette tâche, de même que l'expressivité (cf. intonation des énoncés) pour mimer la folie.

Nous en avons conclu que grâce à ce genre d'oral, l'apprenant peut se libérer de son stress, de sa timidité, enfin de ce qui fait obstacle à la prise de parole devant un public.

Quant à la troisième activité théâtrale, les participants ont été conscients du rôle essentiel de la planification car ils sont sortis du cadre de la classe pour préparer l'espace, les objets nécessaires à la réalisation de la tâche (table, chaises, tablier de cuisine, bouteille d'eau, tasses de café), ont distribué les rôles et appris par cœur les répliques :

68 Extrait $6:$ : La vie d'Albert Einstein »

Scène $1:$ le petit déjeuner

L1 : (frappe les mains) allez Albert allez à table allez / (geste avec la main)

L2 : (court) salut maman

L1 : salut

L2 : (prend la chaise et s'assoit)

L1 : (lui sert du café) (s'assoit) prends ton café × (remue son café)

L2 : (en remuant son café) maman je peux te poser une question /

L1 : oui bien sûr : tu peux mon fils

L2 : pourquoi quand quand je fonds le lait avec ma cuillère (geste avec le pouce) (remue le café) + le sucre se fond rapidement / 
L1 : (hoche la tête à gauche) et toujours la même chose / (plie les doigts et met l'index sur la tempe) (étonnement) tu poses des questions bêtes blizzards où t'as trouvé tout ça (geste avec les deux mains) + moi je vais te répondre Albert / (met le bras droit sur le genou) + bien sûr le sucre se fond rapidement parce que le lait est chaud + tu es convaincu $\uparrow$ (les bras croisés)

L2: non (hoche la tête de gauche à droite) + mais j'ai une autre question (les mains posées sur la table)

L1: c'est mieux de boire ton lait arrêtez de bavarder de bon matin (se lève) tous les =enfants $\times \times \times$ sans s'interroger et alors qu'est-ce que t'as $\uparrow$

L2 : mais maman + n'oubliez pas que Newton il a découvert la loi de la gravité parce qu'il a vu la pomme se tomber sur terre (geste avec la main) alors il a xxx de s'interroger

Le discours produit à l'oral est cohérent. Nous avons relevé quelques syllabes inaudibles, dans l'ensemble, chaque locuteur a pris en compte le dire d'autrui avant d'enchaîner avec sa propre réplique. La stratégie de coopération a donc bien été investie par les participants. Dans toutes les situations, gestes, mimiques et mouvements ont accompagné la parole. La voix était adaptée à la situation de communication (intonation légèrement montante dans la plupart des tours de parole).

Nous avons retenu de ces activités que la forte participation des étudiants, dans les différentes situations de production/interaction orale que nous avons proposées, résultait de leur compétence à mobiliser les stratégies nécessaires à la réalisation de ce genre de tâche. Goullier $(2006$ : 17) explique à ce sujet que «la réussite dans les activités de compréhension ou d'expression, à travers des tâches communicatives, dépend étroitement du développement des compétences qui doit constituer le fil conducteur de la progression ».

De cette expérience, avec les étudiants de licence de français L/LMD, nous avons conclu que la perspective actionnelle peut être exploitée dans l'enseignement de l'oral en Algérie, surtout en ce qui concerne la production et l'interaction orales. Elle est apte à rendre l'apprentissage de la langue étrangère plus efficace parce que plus dynamique. En effet, l'apprenant est en phase avec la réalité qui l'entoure parce qu'elle vise à développer chez lui une compétence à communiquer en combinant le verbal et le non verbal, une aptitude à structurer son discours en s'adressant à l'autre, le libérant des contraintes diverses à l'origine de son mutisme en société. Le recours à ce genre de tâches qui encourage le travail interactif et coopératif en classe de langue étrangère ne peut que contribuer à préparer l'apprenant à assumer un rôle actif dans la société même si, dans son environnement, les interactions en français sont rares.

\section{BIBLIOGRAPHIE}

BEACCO J.-C., L'approche par compétence dans l'enseignement des langues, Didier, Paris, 2007.

BESSE H., Méthodes et pratiques des manuels de langue, Didier / Crédif, Paris, 2003. 
CADRE EUROPÉEN COMMUN DE RÉFÉRENCE POUR LES LANGUES : apprendre, enseigner, évaluer. Unité des politiques linguistiques, Les éditions Didier, Paris, 2001. Disponible sur URL : http://www.coe.int/t/dg4/linguistic/Source/Framework_fr.pdf. Consulté le 06 mars 2016. Coste D., « Tâche, progression, curriculum », in Le Français dans le monde. Recherche et applications, $\mathrm{n}^{\circ} 45,2009$, pp. 15-24.

CUQ J.-P., (Dir), Dictionnaire de didactique du français langue étrangère et seconde, Clé International, Paris, 2003.

CUQJ-P,GRUCAI., Cours de didactique du français langue étrangère et seconde, PUG, Grenoble, 2005. DOLZJ,SCHNEUWLYB., Pour un enseignement de l'oral. Initiation aux genres formels à l'école, (5 ème éd), ESF, Paris, 2014.

GADET F., LE CUNFF C. et TURCO G., (coord), L'oral pour apprendre, Repères, $n^{\circ}$ 17. 1998. Disponible sur URL : http://www.inrp.fr/publications/edition-electronique/reperes/RS017.pdf. Consulté le 20/02/2017.

GARCIA-DEBANC C. et DELCAMBRE I., (coord.), « Enseigner l'oral », in Repères, n 24/25, 2001-2002 (nouvelle série), INRP, Saint-Fons, 2002. Disponible sur URL : [http://www.inrp.fr/publications/ edition-electronique/reperes/RS024-025.pdf]. Consulté le 20/02/2017.

GOULLIER

F., Les outils du Conseil de l'Europe en classe de langue, Cadre européen commun et Portfolios, Les éditions Didier, Paris, 2006. Disponible sur URL : https://www.coe.int/t/dg4/linguistic/Source/Goullier_Outils_1.FR.pdf. Consulté le 27/11/2015.

SHEILS J., La communication dans la classe de langue, Projet $n^{\circ} 12$ : «Apprentissage et enseignement des langues vivantes aux fins de communication ", Les éditions du Conseil de l'Europe, Strasbourg, 1993. Disponible sur le site : [https://www.coe.int/t/dg4/linguistic/ La \%20communication \%20dans \%20la \%20classe \%20de \%20langue \%20web.pdf], consulté le $05 / 11 / 2015$

TAGLIANTE C., La classe de langue, Clé International, Paris, 2007a.

TAGLIANTEC., L'évaluation et le cadre européen commun, (2 2 èmeéd.), Clé International, Paris, $2007 \mathrm{~b}$.

\section{NOTES}

1. Le cursus universitaire LMD (licence/master/doctorat) a commencé à être introduit dans les universités au début des années 2000. Mais il fait l'objet épisodiquement de réajustements sanctionnés par des dispositifs législatifs.

2. Arrêté ministériel $\mathrm{n}^{\circ} 500 \mathrm{du} 28$ juillet 2013. Disponible sur le site du ministère de l'enseignement supérieur et de la recherche scientifique : <https://www.mesrs.dz>

3. Un module de 3 ou 4 heures consacré à la fois aux techniques d'expression écrite et orale (T.E.E.O.), en première année d'une licence qui en comptait quatre (4).

4. Pour Francine Cicurel (2011: 24), « au cours d'une interaction donnée, on peut supposer que les protagonistes vont "parler de quelque chose ", partager un thème conversationnel. (...) Il n'est pas simple de déterminer quel est le "thème" d'un cours de langue, car tout est susceptible d'être objet de discours: un thème de société au cours d'une discussion. (...) c'est l'enseignant qui impose le thème, l'annonçant le plus souvent ».

5. Conventions de transcriptions du Groupe Aixois de Recherche en Syntaxe (GARS), sous la direction de C. Blanche-Benveniste. Voir, en annexe, les signes de transcription utilisés. 
6. Il s'agit d'un reportage intitulé "Des femmes dans des métiers masculins », diffusé par une chaîne française et disponible sur le site: http://fr.ver-taal.com/ jt_20101127_femmesmetiersmasculins.htm.

7. Cette échelle comprend six niveaux : A1, A2, B1, B2, C1, C2.

8. Tagliante (2007b: 48-50) reprend le classement des compétences adopté dans le CECRL en insistant sur le fait que la compétence à communiquer langagièrement est constituée de plusieurs composantes : linguistique, sociolinguistique, pragmatique et fonctionnelle.

9. [jak] : Ah bon !

10. [flen] : Quelqu'un

11. [flena] : Quelqu'une

12. [jalala] : expression de l'arabe qui signifie « majesté ».

\section{RÉSUMÉS}

Le présent article est la synthèse d'une expérience effectuée à la section de français du département des lettres et langues étrangères, de l'université de Tiaret, en Algérie. Elle porte sur l'enseignement / apprentissage de la compétence de production orale et met en pratique quelques-uns des principes de l'approche actionnelle à travers l'étude des interactions orales au cours de la réalisation d'une tâche langagière.

Notre objectif est d'amener les apprenants à mobiliser de multiples ressources lors de la prise de parole en classe de FLE. Il s'agit de mettre à l'épreuve l'un des principes théoriques qui soustendent la conception de la langue dans le Cadre Européen Commun de Référence (Conseil de l'Europe, 2001), à savoir celui de l'apprenant comme «acteur social » ayant des tâches à accomplir tout comme dans la vie réelle.

Notre corpus oral a été constitué d'enregistrements filmés de séances d'expression orale avec les étudiants de $2^{\text {ème }}$ année de licence du dispositif LMD.

The present article is the synthesis of a research carried out in the French section of the Department of Letters and Foreign Languages, at Tiaret University, Algeria. It focuses on the teaching/learning of oral production skills and practices some of the action-oriented approach principles through the study of oral interactions during the performance of a language task. The aim behind this is to lead the learners to mobilize multiple resources when speaking in FFL (French as a Foreign Language) classes. It is a question of testing one of the theoretical principles underlying the conception of language in the Common European Framework of Reference (Council of Europe, 2001), namely the fact that the language the learner is regarded as a "social actor" having tasks to perform just similar to real-life situations. The corpus consists of filmed recordings of oral expression sessions conducted with second year LMD students. 
INDEX

Mots-clés : Cadre européen commun de référence, approche actionnelle, tâche, compétence orale, interaction orale, stratégie d'apprentissage

الإطار المرجعي الأوروبي المشترك, مقاربة عملية, مهمة, كفاءةفهرس الكلمات المفتاحية: شفوية, تفاعل شفوي, استراتيجية التعلم

Keywords : Common european framework of reference, action-oriented approach, task, oral competence, oral interaction, learning strategy

\section{AUTEUR}

\section{HAMIDA DOULATE SEROURI}

Université Abdelhamid Ibn Badis Mostaganem - Algérie 